\title{
MedienPädagogik
}

www. medienpaed.com

Zeitschrift für

Theorie und Praxis

der Medienbildung

ISSN 1424-3636

\section{«Hallöchen Herr Professor!» \\ Überlegungen zur Normierungsproblematik in der E-Mail-Kommunikation am Beispiel des Hochschulkontextes}

Nicole Hoffmann, Katrin Keller und Anke Pfeiffer

\begin{abstract}
Obwohl die E-Mail mittlerweile zu den alltäglichen Kommunikationsformen an Hochschulen zählt, kommt es immer wieder zu Irritationen - ausgelöst durch E-Mails, die von Seiten der Lehrenden zum Teil als «schlampig» oder "frech» empfunden werden. Dieses Phänomen wird im vorliegenden Beitrag zum Anlass genommen, der Frage nachzugehen, warum sich in der E-Mail-Kommunikation bislang wenig allgemeingültige Regeln herausgebildet haben.

Dabei wird erstens die These vertreten, dass die (oftmals gescholtenen) studentischen E-Mails keineswegs regelfrei verfasst werden; sie werden aber von den Empfänger/innen als "abweichend» erlebt, da die E-Mail als Gattung oder Textsorte - insbesondere zu Beginn der Kommunikation - auf sehr unterschiedlichen Referenztextsorten fusst: Die Verfasser/innen orientieren sich an zahlreichen anderen Formaten, wie etwa dem Brief, der Kurznotiz, dem Alltagsgespräch, der Verfahrensanweisung oder dem Kommentar. Erst im Verlauf einer weiteren E-MailKommunikation können dann gemeinsame Regeln ausgehandelt werden. Da jedoch - so die zweite These im Anschluss - der E-Mail-Verkehr meist interpersonal geschlossen bleibt, kommt es nicht zu einer kollektiven Normbildung, die durch grössere Öffentlichkeiten dauerhaft geteilt würde. Solange also die Referenznormen zu Beginn eines Austausches derart unterschiedlich sind, und sie für die jeweilige digitale Interaktion im Verlauf immer neu interpersonal justiert werden müssen, solange wird die E-Mail als ein individualisierender, vermeintlich regelloser «Selbstläufer» wahrgenommen. Die beiden Thesen basieren nicht auf eigenen empirischen Erhebungen, sie stellen vielmehr Überlegungen dar, die das alltäglich auftretende Phänomen der "Normverletzung» bei E-Mail-Texten - methodisch explorativ anhand von Beispieltexten - aufgreifen, um es mit Forschungsbefunden zur Spezifik der E-Mail bzw. zur digitalen Kommunikation zu kontrastieren bzw. zu erschliessen.
\end{abstract}




\section{Problematisierende Einleitung}

«Hallöchen Herr Professor!» oder «Hier flattert meine Präsi durch die Leitung!», solche Sätze sind in E-Mails an Hochschulen keine Seltenheit. Und immer wieder werden die teilweise kryptischen, bruchstückhaften oder umgangssprachlichen Mails ${ }^{1}$ als «unhöflich», «formlos» oder «unmöglich» kritisiert. So entwirft beispielsweise Thomas Hoeren, Professor für Informations- und Telekommunikations- und Medienrecht an der Universität Münster, ein «E-Mail-Gruselkabinett». Er gruppiert die erhaltenen studentischen Mails nach unterschiedlichen Typen, wie u. a. der Typ «Babelfisch» (Zitat: «Weil Prufung war ganz schwierig») oder der Typ «Feldwebel» (Zitat: "Gehe von Erledigung aus»). Hoeren sieht sich in einem "Dschungel von Pseudo-Anbiederei, Jugend-Slang und Hybris» «fernab der geregelten Bahnen universitärer Kommunikation und brieflichen Anstandes» (Hoeren 2006).

Offensichtlich kollidieren hier die Erwartungen, die von den Empfänger/innen an das angemessene Verfassen einer Mail gestellt werden, mit den Auslegungen des sprachlichen Möglichkeitsspektrums, das in den Augen der (studentischen) Verfasser/innen als zulässig gilt. Entsprechend tauchen seit Jahren immer wieder Forderungen nach einer 'Netiquettes auf, die - etwa in Anlehnung an Knigge - einen Verhaltenskodex für die Kommunikation in Internet und Mail proklamieren (vgl. u. a. Mai 2007 oder Jörn 2008). Doch trotz Normierungsanliegen und Standardisierungsvorschlägen konnten sich bislang keine allgemeingültigen Schemata durchsetzten (vgl. u. a. Mai 2007; Döring 2003; Dürscheid 2009).

Warum folgt die E-Mail-Kommunikation - etwa zwischen Studierenden und Lehrenden - kaum einheitlichen bzw. konsensfähigen Regeln? Warum wird die E-MailPraxis weiterhin problematisiert, obwohl das Medium schon seit vielen Jahren Bestandteil der gängigen Kommunikationspraxis an Hochschulen ist?

Im Folgenden wird - anhand von in qualitativ-explorierender Absicht ausgewählten E-Mail-Text-Beispielen aus dem Hochschulalltag - der Versuch unternommen, das Phänomen der sprachlichen Heterogenität von Mailtexten bei gleichzeitig normativen Standardisierungserwartungen aus der spezifischen sozialen Praxis des Mail-Verkehrs heraus zu verstehen. Dazu wird zunächst ein Blick auf markante Merkmale der E-Mail als Textsorte bzw. als Kommunikationsform geworfen (2.), um in einem zweiten Schritt speziell die Normierungsproblematik zu erschliessen (3.). Hierzu werden zum einen das Gattungsverständnis zu Beginn des Mail-Verkehrs (3.1) und zum anderen der weitere Verlauf der Kommunikation mit Bezug auf die Bedeutung von sozial vermittelten Aushandlungspraktiken und dauerhaften Öffentlichkeiten (orientiert am «social identity model») (3.2) berücksichtigt. Den Abschluss bildet ein zusammenfassendes Fazit (4.).

1 Im Weiteren werden die Begriffe E-Mail und Mail synonym verwendet. 


\section{Zur Spezifik der E-Mail als Textsorte bzw. als Kommunikationsform im Hochschulkontext}

Die Geburtsstunde der E-Mail-Kommunikation verläuft in Deutschland nahezu zeitgleich mit der Nutzung des Internets im Allgemeinen. Zu den ersten Protagonist/ innen vor gut 25 Jahren zählen studentische Aktivist/innen an den Universitäten in Karlsruhe und Dortmund; von dort wurden die ersten digitalen Vernetzungen vorangetrieben. Von da an etablierte sich «die E-Mail» zunehmend als Kommunikationsmedium; innerhalb von zwei Jahrzehnten avancierte sie vom belächelten Aussenseiter für PC-Freaks zu einem Leitmedium der schriftlichen Kommunikation (vgl. Zuber-Knost 2009).

Einer Studie von Middendorff aus dem Jahr 2002 zufolge gaben bereits damals $87 \%$ der Studierenden an, das E-Mail-Format zu nutzen. Neben der Textverarbeitung und dem allgemeinen Umgang mit PC und Internet gehörte der Mail-Verkehr zu den technisch am besten beherrschten Anwendungen bei Studierenden. Darüber hinaus zählte die Mail-Kommunikation zu denjenigen PC-Nutzungsmöglichkeiten, denen die höchste Relevanz im Studium beigemessen wurde (vgl. Middendorff 2002, 5 ff.). Im Jahr 2011 ist das Studieren ohne einen Mail-Zugang nahezu unmöglich geworden. Wichtige Teile der Lehrveranstaltungs-, der Prüfungs- und der Studienorganisation werden über digitale Verwaltungssysteme abgewickelt, die für Studierende einen Mail-Account unumgänglich machen.

Die elektronische Post ist dabei eingebunden in einen viel umfangreicheren Prozess der Digitalisierung der Lebenswelt im Allgemeinen bzw. des Einzugs neuer Medien an den Hochschulen im Besonderen. Ihre Einordnung in das aktuell viel diskutierte Web 2.0 fällt hierbei unterschiedlich aus. Fischer und Gscheidle (2008) zählen die E-Mail zu den Vorläufern der Web-2.0-Instrumente. Sie eröffnete Studierenden von Beginn an die Möglichkeit zu Partizipation und Informationsweitergabe sowie zu Kontaktaufnahme und Austausch mit Kommiliton/innen und Lehrenden (vgl. Fischer/Gscheidle 2008). So integrieren Web-2.0-Formate, wie Blogs, Wikis oder Podcasts, in der Regel die E-Mail-Funktion bzw. an die EMail angelehnte Funktionen u. a. in Kommentar- oder Diskussionsforen. Trotz aller Nähe sieht Schulmeister die Mail jedoch primär als «Kommunikationsmethode», die nicht dem Bereich der Web-2.0-Tools zuzuordnen ist (vgl. Schulmeister 2010). Auch in der Studie «Studieren im Web 2.0» von Kleinmann et al. (2008) findet die E-Mail keine Berücksichtigung. So verweisen Kesselheim und Lindemann (2010) darauf, dass die E-Mail - im Gegensatz zu Weblogs, Wikis oder Portfolios - im studentischen Lernalltag zwar kaum Erwähnung findet, aber doch allgegenwärtig ist. Die E-Mail hat sich an den Hochschulen als Bestandteil der gängigen Kommunikationspraxis etabliert - und das gilt auch für die Seite der Lehrenden bzw. Forschenden. So portraitiert Grigat den diesbezüglichen Berufsalltag eines «Akademikers» mit den Worten: «Die E-Mail ist für ihn eine Katastrophe (...). Dennoch, und das 
gehört zu dieser fatalen Dialektik, liebt er die E-Mail auch - irgendwie. Er schätzt es ja, dass er seine Kollegen mal eben etwas fragen oder mitteilen kann - ob diese nun in Adelaide oder auf der anderen Seite des Flurs an ihren Schreibtischen sitzen. Das ist nützlich, aber deshalb auch ein Problem. Denn leider haben dies auch die Kollegen erkannt - und die Verwaltung, die Freunde und die Studenten. Und deshalb wiederum hat für ihn die Email ihre Unschuld verloren und ist zu einem richtigen Biest mutiert. Es lässt sich nicht abwimmeln, hat einen grossen Appetit und frisst Zeit und Aufmerksamkeit. So bekommt jeder Schreibtischmensch durchschnittlich 74 E-Mails pro Tag (davon 61 erwünschte) und 13 Spam-E-Mails). Er verschickt im Schnitt 36 pro Tag. Gefühlt sind es oft viel mehr» (Grigat 2010, 646). Aus Sicht der Forschung wird der Erfolg der Mail unter anderem darauf zurückgeführt, dass sie orts- und zeitunabhängig eingesetzt werden kann und als besonders schnelles Medium der Datenübertragung gilt. Doch nicht nur die einfache Anwendung von E-Mail-Programmen dient ihrer weiten Verbreitung; die Mail scheint anschlussfähig, da sie als «soziotechnisches System den funktionalen Kommunikationsbedürfnissen der Anwender nachkommt» (Schelske 2005, 143). So hat mit der E-Mail ein besonderes bzw. spezifisches Kommunikationsformat Einzug gehalten: In einer Kategorisierung zählt Emmer die Mail dabei zu den asynchronen Online-Medien, die sich trotz Ungleichzeitigkeit durch eine hohe Interaktivität auszeichnen. In der Regel findet der Kontakt «one/few-to-one/few» statt, also eine/einer und/oder wenige kommunizieren mit einer/einem und/oder wenigen. So betrachtet, gestaltet sich das Versenden und Empfangen von Mails als ein individuelles Geschehen. Sie ist kein anonymes Medium, sondern dient der gezielten Konversation, die - gerade im Hochschulkontext - in den meisten Fällen mit einem gezielten Anliegen verbunden ist (vgl. Emmer 2005). Döring zufolge übernimmt die E-Mail hier hauptsächlich die Kommunikationsaufgaben: Informieren, FragenStellen oder In-Verbindung-Bleiben (vgl. Döring 2003). Zwar werden Mails auch in Newsgroups, Lernplattformen oder elektronischen Gästebüchern geschrieben, über Mailinglisten an eine grosse Zahl von Empfänger/innen verschickt oder sind als Kommentare in Weblogs zu lesen, doch bleibt der Zweig der interpersonalen, damit eher nicht-öffentlichen Kommunikation prägend für die Beschreibung der Mail als Medium (vgl. Dürscheid 2006).

«Fast alles ist möglich», lautet auch das Fazit von Schmitz zur E-Mail-Kommunikation; er verweist auf eine nahezu unerschöpfliche Bandbreite an Ausdrucksformen dieser computervermittelten Verkehrsform, die Privatheit und Öffentlichkeit, Spontaneität und Sorgfalt sowie Sprechbarkeit und Schreibbarkeit zulässt und verbindet (Schmitz 2002, 33). Das Spektrum reicht von schnell <ins Unreine getippten Gedankenfetzen> bis hin zu elaboriert ausgefeilten, hoch formal strukturierten Schriftstücken. Die E-Mail weist somit Elemente mündlicher und schriftlicher Kommunikation auf; sie wird hier eher als beziehungsbezogene Kommunikationsform 
verstanden und weniger als Textsorte oder als spezielle schriftliche Gattung (vgl. auch Ziegler 2002).

Als Kommunikationsform betrachtet hängt die Art der Äusserung zum einen von den technischen Bedingungen des Mediums ab sowie stärker noch von den $\mathrm{Be}$ dingungen der einzelnen Kommunikationssituation. Dazu zählen Rollen- und Persönlichkeitsmerkmale der Kommunikationspartner/innen bzw. deren soziale Beziehung. Für den Hochschulkontext von Bedeutung sind demnach der Bekanntheitsgrad zwischen Studierenden und Lehrenden, die Interpretation der sozialen Hierarchie, persönlicher oder beruflicher Bezugspunkt der Kommunikation bzw. Anliegen oder Zwecke einer Mail. "Gerade der persönliche Lebensstil, das Alter, der Bildungsgrad, der Beruf, die Interessen, die Lebenslage und damit auch das Netz der Kommunikationspartner, des Mail-Verfassers bestimmen die Zwecke, Themen, Inhalte und (grösstenteils in deren Folge) auch die sprachlichen Formen in einem ganz erheblichen Masse» (Schmitz 2002, 41). Insofern überrascht es nicht, dass Mails ein breites Spektrum stilistischer Vielfalt abbilden.

Aber auch die immer wieder in der Forschung herangezogene Referenzform des klassischen Briefs ist von Persönlichkeitsmerkmalen, Schreibanlässen und Statusfragen geprägt. Generell zeigen sich im Vergleich von Brief und E-Mail zahlreiche Ähnlichkeiten, aber auch vielfältige Abweichungen, die in den gängigen Textstrukturmerkmalen, wie Anrede, Textkörper und Grussformel, sichtbar werden. So führt Pansegrau an, dass im Vergleich zum herkömmlichen Brief in E-Mails die üblichen Anreden kreativ und situativ gestaltet werden (vgl. Pansegrau 1997). Des Weiteren weist er darauf hin, dass E-Mails häufig sogar dann Anrede und Verabschiedung beinhalten, wenn sich der Inhalt auf einen einzigen Satz beschränkt. In diesen Fällen wird die rahmende Nähe des Brief-Formats überdeutlich. Auch Dürscheid vergleicht in ihrer Forschung die Mail mit dem klassischen Brief: Im Unterschied zum herkömmlichen Brief würden in der Mail jedoch tendenziell mehr Möglichkeiten der sprachlichen Gestaltung ausgeschöpft. In ihren Untersuchungen konnte sie deutlich machen, dass E-Mails sowohl Brief- als auch Gesprächscharakter besitzen. Sie bezieht sich dabei auf Elspass, der darauf verweist, dass mit der E-MailKommunikation keine neuartige Sprachgebrauchsform entstanden sei, sondern vielmehr eine veränderte und damit neue Sprachgebrauchsnorm (vgl. Dürscheid 2009).

Ein weiterer Unterschied zeigt sich auf der Bedeutungsebene. Briefe gelten auch heute noch häufig als Zeichen der Verbundenheit, ihnen werden tendenziell Attribute wie Besonnenheit, Privatheit und Zuverlässigkeit zugesprochen. Dagegen wirkt die E-Mail oft eher unpersönlich. Sie wird mit geringerem Aufwand verfasst, ist schnell verschickt und $u$. $U$. rasch zu lesen. Sie verführt Verfasser/innen, jede noch so belanglose Kleinigkeit zu versenden, die im Moment aber von Relevanz zu sein scheint. Die schriftliche Kommunikationsschwelle wird herabgesetzt, und da- 
mit öffnet sich ein neues Feld, das eben nicht im Sinne des sorgfältig entworfenen Briefs reglementiert ist (vgl. Meier 2002). So werden E-Mails zum Teil eher als Gebrauchstexte verstanden. Mündlichen Sprachäusserungen entsprechend gleichen sie dann stark situationsbezogenen, konkreten Sprechakten (vgl. Dürscheid 2006; Günther/Wyss 1996).

\section{Zur Normierungsproblematik im E-Mail-Verkehr an Hochschulen}

Setzt demnach mit dem verstärkten Aufkommen der E-Mail-Kommunikation an Hochschulen eine breit gefächerte Diversifikation des schriftlichen Austauschs zwischen Lehrenden und Studierenden ein, so muss diese mögliche Vielfalt an Erscheinungsformen noch kein grundsätzliches Problem darstellen. Sie wird erst dann problematisch, wenn unterschiedliche Vorstellungen davon aufeinanderprallen, wie eine "gute» Mail auszusehen hat.

Entgegen ihrer Charakterisierung in der bisherigen Forschung als strukturell facettenreicher Kommunikations- bzw. Interaktionsform mit Merkmalen von Schriftlichkeit und Mündlichkeit scheinen sich jedoch im Alltag immer wieder Konflikte an der Wahrnehmung der E-Mail als einer mit bestimmten, erwartbaren Merkmalen assoziierten Textsorte zu entzünden. In den langen Jahren ihres Gebrauchs hätten diese Erwartungen an die Mail als Textsorte inzwischen in genormten Konventionen münden können. Es hat sich jedoch (bislang) kein konsensuelles Regelsystem etabliert, das sich etwa am klassischen Briefverkehr, an der Netiquette oder an anderen Kriterien orientiert (vgl. auch 1.).

Dieser Erhalt der Heterogenität bei gleichzeitiger Normierungserwartung kann aus den spezifischen Verkehrsbedingungen der E-Mail als sozialer Praktik gedeutet werden. D. h. im Unterschied zu Kommunikationsmedien, die faktisch oder potenziell eine stärkere Öffentlichkeitsorientierung aufweisen, zeigt sich, dass der erwartete Normierungsprozess bei der E-Mail-Kommunikation zum ersten von unterschiedlichen Referenzpunkten ausgeht (3.1) und zum zweiten im Anschluss einen anderen Verlauf nimmt bzw. anderen Bedingungen unterliegt (3.2).

\subsection{Normgebende Referenzen zu Beginn der E-Mail-Kommunikation}

Mag die professorale Klage der kommunikativen Unangemessenheit studentischer Mails wegen der beklagten Normverstösse vorschnell dazu verführen, diese Mails als «ungeregelt» zu betrachten, so sind sie dies bei näherer Betrachtung keineswegs. Sie schöpfen vielmehr aus einer grossen Bandbreite von Textsorten bzw. Sprachformen. Die Verfasser/innen von Mails greifen dabei zu Beginn einer Kommunikationseinheit jedoch auf Formate zurück, deren Normen aus anderen, online wie offline existierenden, schriftlichen oder mündlichen Kontexten stammen. So lässt sich beispielsweise eine stilistische Orientierung an folgenden Referenzformen ausmachen: Brief, Telegramm, SMS, informierende Notiz, Nachtrag, 
Begleitschreiben, Chat, Formblatt, Meldung bzw. Zwischenbericht, Verfahrensanweisung, Kommentar, Bewerbung, Bitt- und Dankesschreiben, Entschuldigung, Tür-und-Angel-Gespräch, quasi-gesprochene Beantwortung einer Frage, anekdotisches Erzählen, Selbstreflexion oder Gedankenstrom. Wie sich der Bezug auf unterschiedliche Referenztexte auf die Formulierung der Mail auswirkt, verdeutlichen die folgenden Beispiele aus dem Hochschulkontext: ${ }^{2}$

Referenzform «Begleitschreiben»:

«Sehr geehrte Frau Kuhn,

anbei meine Präsentation mit den Literaturangaben.

Mit freundlichen Grüssen

Eva-Maria Henrich»

Referenzform «informierende Notiz»:

«Der Link zu: Rogge, Jan Uwe: Das_find'_ich_total_spannend kann nicht geöffnet werden. Fehlermeldung: defekt.

LG

Stefan Jakobs»

Referenzform «Verfahrensanweisung»:

«bitte drucken. Wobei HEX_ZWN_Auswertung_5 und HEX_ZWN_Auswertung_5_1 bitte

in bunt drucken. Die Excel Datei ist noch auf kein "gutes" Druckformat

bzw. Druckbereich eingestellt. Könnten Sie das noch passend einstellen?

Das wäre super. Danke.

Frieda Biener»

Referenzform «Beantwortung einer Frage» oder «mündliche Anschlusskommunikation»:

«Nein, bringe ich direkt vorbei. Wäre nächster Montag ok?»

Referenzform «alltägliches Tür-und-Angel-Gespräch»:

«Hi,

wollt nur meine Texte loswerden :-) ... schönen Abend und bis bald

Liebe Grüsse, Sabine»

Referenzform «Meldung» oder «Zwischenbericht»:

«Hallo,

2 Bei den Belegbeispielen handelt es sich stets um die vollständige, wenn auch anonymisierte Wiedergabe des jeweiligen Mailtextes. 
da heute der 14.4. ist, möchte ich mich mal zurück melden.

Ich kann noch keine neuen Dokumente rumschicken, da ich bisher nur Teilergebnisse bekommen habe.

Ja, soviel zum Stand der Dinge.

Oliver»

Referenzform «erzählende Selbstreflexion»:

«Und wegen Termine: Ich kann immer ab 16.00 Uhr. Bis auf: 10.07. - 24.07. da weile ich auf Gomera und quäle mich sowohl mit der Hitze als auch mit Textanalyse.

Habe ja im Moment so was wie Muffensausen, nach dem Motto: Ich könnt ja dann mal mit der eigentlichen Grounded Theory-Arbeit anfangen ...

Denke dauernd so Sachen wie: Und wenn ich jetzt falsch analysiere? Dann ist die ganze Arbeit im Eimer. Nennt man wohl Angst vor der eigenen Courage, oder?

Und dann sind da noch so Gedanken wie: Oh Gott, ist das viel Arbeit! Will mit 'nem Text anfangen, der neun Seiten lang ist ... Und den ganzen Text nehmen, allein zu Übungszwecken und um mal den Start zu machen.

Einen frohen Tag im Kreisssaal des Wissens!

LG, Sue

Ach ja: Gruss an die anderen Zwei ... oder Drei!»

Referenzform «Telegramm» oder «SMS»:

«bin gleich da - lg - M»

Referenzform «lautes Denken» oder «Gedankenstrom»:

«nochmal zu dem Thema Theater an Schulen. Es findet an Schulen statt, aber nicht mit Personen, die dafür ausgebildet sind. DAs will man ändern. DAS Minsiterium hat die Iniitiative von d en Praktikern im LAnd aufgegriffen und standards dafür gemacht. (Claus war auch dabei) DAs heisst, als WAHL, nicht flächendeckend. War mir noch ein Bedürfnis, gerade Dir das mitzuteilen.»

\subsection{Normenbildung im weiteren Verlauf der E-Mail-Kommunikation}

Ist die digitale Kommunikation zu Beginn einer Sequenz oftmals von Bezügen auf die Regeln anderer Praktiken bzw. -formen des mündlichen wie des schriftlichen Austauschs geprägt, so kann sie im weiteren Verlauf durchaus eine spezifische Normierung erfahren.

Auch Matzat (2002) stellt im Hinblick auf die Entstehung von Normen in akademischen Online-Gruppen fest, dass das Kommunikationsverhalten der Mitglieder 
häufig durch Netzwerke sozialer Beziehungen, die auch offline existieren, beeinflusst ist. Unter Bezug auf Coleman verweist er weiter darauf, dass die Geltung einer Norm kein Merkmal eines Individuums sei, sondern im Prozess einer Gruppe ausgehandelt werde. Für die Entstehung des Bedürfnisses nach Normen müsse eine (kommunikative) Handlung eines Gruppenmitglieds die Interessen der anderen Mitglieder tangieren, d. h. es besteht ein wie auch immer gearteter Regelungsbedarf, der nicht anderweitig zu bearbeiten ist. Weigert sich ein Gruppenmitglied, auf die Ausübung seiner individuellen Handlungsrechte zu verzichten, können darauf Sanktionen folgen. Von den Wirkungen der Sanktion sollte i.d.R. jedes Gruppenmitglied profitieren, unabhängig von seinem Einsatz für die Umsetzung der Norm. Normeinhaltung und Sanktionierung bedingen sich damit gegenseitig. Eine hohe Beziehungsdichte vereinfacht die Ausübung von Sanktionen durch organisiertes gemeinsames Handeln (vgl. Matzat 2002). Damit gewinnen die Öffentlichkeit der Gruppe und die Art der Austragung der Beziehungsregelung an Bedeutung für die Entstehung von (hier medien-) spezifischen Normen.

Der Entstehung von Normen in netzbasierten Medien gilt auch das Interesse von Postmes, Spears und Lea (2000), die sich u.a. mit der Analyse virtueller studentischer Communities beschäftigten. Sie stellen ebenfalls fest, dass Normen dort aktiv ausgehandelt werden und der Normentstehungsprozess davon beeinflusst wird, wie die Gruppenmitglieder zueinander stehen. Für Postmes, Spears und Lea reicht dies bis hinein in die Entstehung einer sozialen Identität der Gruppe. Sie gehen von der Annahme aus, dass virtuelle Gruppen für ihre Mitglieder durchaus sehr real sein können, obwohl wenig oder kein persönlicher Kontakt stattfindet. D. h. selbst bei absoluter Anonymität ist ein regelhaftes Verhalten und eine gewisse Verbindlichkeit den anderen Mitgliedern gegenüber nicht ausgeschlossen; im Gegenteil, so ihre These, auch virtuelle Gruppen müssen ein bestimmtes Repertoire an Normen herausbilden, um als Gemeinschaft agieren zu können. Im Sinne des «social identity models» entstehen Normen durch aktive Kommunikations- und Aushandlungsprozesse der Gemeinschaft, in deren Verlauf die Akteur/innen zunächst die Praktiken anderer beobachten. Im Laufe der Zeit neigen die Mitglieder einer virtuellen Gruppe dazu, bestimmte als gruppentypisch erlebte Kommunikationsformen anzunehmen, sie zu verinnerlichen und sich entsprechend zu verhalten. Der Einfluss der Gruppe entscheidet demnach darüber, wie ein Medium prinzipiell zu nutzen ist. Gelingt die Normenbildung, ist jede Äusserung sowohl ein Produkt des Individuums als auch der Gruppe (vgl. Postmes/Spears/Lea 2000).

Diese Befunde zu anderen digitalen Kommunikationsformen lassen sich einerseits auf die Analyse der Normierungsproblematik bei E-Mails übertragen - insofern als erstens prinzipiell eine dialogische Situation bzw. die Möglichkeit der Rückmeldung gegeben ist. Denn um eine Regelhaftigkeit innerhalb der Mail-Kommunikation erkennen, verstehen und sich gegebenenfalls auf Normen einigen zu können, 
bedarf es einer Rückmeldung (vgl. auch Schulmeister 2006). Dies bedeutet zweitens, dass der wechselseitige Kommunikationsprozess länger andauern muss, um eine Aushandlung von Regeln zu ermöglichen. Schelske (2005) verweist dabei auf einen Unterschied zur synchronen Kommunikation: Wie oben bereits erwähnt wird die Mail als «schnelles` Medium gehandelt, d. h. die schriftliche Kommunikation erfährt eine Beschleunigung, da innerhalb weniger Sekunden die Übertragung von Daten jeglicher Art stattfinden kann. Dagegen wird sprachliche Kommunikation verlangsamt bzw. entschleunigt. In Folge dessen dauert der Aufbau sozialer Beziehungen bzw. Normen zwischen Verfasser/innen und Empfänger/innen länger (vgl. Schelske 2005).

Andererseits zeigt die übliche E-Mail-Praxis einen markanten Unterschied zu den o.g. Online-Gruppen oder Communities mit einer geteilten Öffentlichkeit, denn selbst wenn es im Prozess einer längeren Kommunikationssequenz gelingt, sich auf bestimmte Regeln zu verständigen, so bedeutet dies im Fall der E-Mail, dass es sich meist um eine Dyade, um eine kleine, geschlossene "Gruppe» handelt. In der Kommunikation mit anderen Mail-Partner/innen kann die Aushandlung parallel einen ganz anderen Verlauf nehmen. Damit unterscheidet sich die Mail in der Normierungsfrage auch vom klassischen Brief. Bestimmte Form- und Stilelemente des Briefs konnten sich als allgemein ‘angemessen` durchsetzen, da die Briefform insbesondere im 18. und 19. Jahrhundert auch als öffentliches Medium verstanden wurde. Der Brief war oftmals zum Vorlesen innerhalb eines interessierten Kreises konzipiert oder gar als Briefroman oder wissenschaftlicher Briefwechsel zur Publikation gedacht. Diese geteilte Öffentlichkeit bzw. dieser gesellschaftliche Anspruch fehlt den Aushandlungsbemühungen des E-Mail-Verkehrs jedoch in den meisten Fällen. Zugespitzt verweist Vedder (2010) exemplarisch auf den Universalgelehrten Albrecht von Haller, der im 18. Jahrhundert mit seinen etwa 17.000 Briefen ein ausgedehntes Korrespondenznetz schuf - und sie fragt: «aber wer wird je 17000 E-Mails als wissenschaftshistorische Quelle edieren?» (Vedder 2010, 637). So bleibt die E-Mail bislang im Normalfall ein individualisiertes Medium des alltäglichen Gebrauchs: vom Beginn der Entscheidung der Verfasser/innen angesichts der vielfältigen möglichen Gestaltungs- und Ausdrucksvarianten, über Wahrnehmung bzw. Bewertung und vor allem Reaktion der Empfänger/innen und ggf. bis hin zur längerfristigen Herausbildung bestimmter Normen in der computervermittelten Kommunikation zwischen diesen Partner/innen.

Wie sich die Aushandlung von Regeln im Laufe des E-Mail-Verkehrs zwischen Studierenden und Dozierenden entwickeln kann, soll anhand des folgenden anonymisiert wiedergegebenen E-Mail-Dialogs aus dem Wintersemester 2009/2010 exemplarisch verdeutlicht werden: 
Eine Dozentin richtet sich mit weiterführenden Informationen zur Anfertigung einer Seminararbeit an eine der Seminar-Arbeitsgruppen.

Dozentin:

«Liebe AG3,

vielen Dank für die Überarbeitung des Themas. Leider muss ich Sie noch um einige Korrekturen bitten. $(\ldots)^{3}$

- Die Internetverweise sind zu ungenau. Sie sollten hier auf konkrete Seiten verweisen und nicht auf komplette Homepages (z. B. www.uni-saarland.de hat nichts mit dem Begriff zu tun).

- Die Seite www.schulratgeber.de kann nur sals Mitglied komplett geladen werden. Diese Seite bitte entfernen und eine andere Quelle nutzen. Übernommene Texte überarbeiten. (...)

- Bitte setzen Sie auch «Interne Verweise` auf Begriffe, die bereits im Glossar vorhanden sind. (...)

Falls Sie noch Fragen dazu haben, können Sie sich jederzeit gerne bei mir melden.

Beste Grüsse

Andrea Bach»

Frau Bach nennt hier im Kern die entsprechenden Arbeitsanweisungen. Ihr Stil ist höflich konkretisierend; er entspricht im Rahmentext der klassischen Briefform mit Begrüssungs- und Abschiedsformel sowie einleitender Bezugnahme und nimmt zugleich Elemente der «Verfahrensanweisung» auf.

Eine Studentin übernimmt nun vermittelnd den Schriftverkehr zwischen der Dozentin und ihrer Arbeitsgruppe.

Studentin:

«Hallo,

wenn ich auf den Link des Schulratgebers gehe (und nicht nur ich), dann kommen genau die Informationen auf der Seite die wir benutzt haben ohne irgendwelche Passwörter zu benutzen oder so. Bitte schauen sie da nochmal nach.

Tanja Bender»

Die Studentin startet mit einem kurzen «Hallo» ohne persönliche Anrede; ihre Mail entspricht formal eher einem «lauten Denken» bzw. einer Art des «kommentierenden Gedankenstroms». Die Studentin fordert die Dozentin auf, Angaben noch-

3 An dieser Stelle und im weiteren Verlauf werden aufgrund der Ausführlichkeit der Angaben der Dozentin nur die signifikanten Passagen des Mail-Verkehrs wiedergegeben. 
mals zu überprüfen, wobei die fehlenden nonverbalen oder paraverbalen Kommunikationsaspekte den Text u. U. «forsch» erscheinen lassen können.

Die Antwort der Dozentin lässt davon aber zunächst nichts merken - und sie beharrt auf der Briefform:

Dozentin:

«Liebe Frau Bender, danke für Ihre Rückfrage.

Bei mir taucht die folgende Seite auf: (...)

Bitte geben Sie mir nochmals die korrekte Adresse an.

Beste Grüsse

Andrea Bach»

Auch die Studentin bleibt bei der eingangs von ihr gewählten Form:

Studentin:

«Wenn sie den Link anklicken?

Ausserdem haben wir leider keine Begriffe in unserem Skript, die wir auf schon bestehende interne verweisen könnten.

Tanja Bender»

In der folgenden Antwort versucht die Dozentin nun, den inhaltlichen Strang fortzusetzen, ergänzt die Mail jedoch noch um eine regel-thematisierende bzw. kritisierende Metakommunikation an die Studentin selbst:

Dozentin:

«Hallo Frau Bender, liebe AG3,

da Sie innerhalb des Fliesstextes die Hauptseite eines Internetratgebers (...) angegeben haben, war es für mich nicht gleich nachvollziehbar, auf welche Seite sie sich der jeweilige Abschnitt bezieht. (...)

Beste Grüsse

Andrea Bach

P.S. @Frau Bender: Ich hoffe, dass wir diese Zusammenarbeit im Rahmen des Seminars in einem höflichen Umgangston abschliessen können. Auch als Ratschlag für die Zukunft: Bedenken Sie, wie ihre Mail (die Sie ja stellvertretend für ihre Gruppe gesendet haben) beim Empfänger ankommt.»

Ohne auf die persönliche Ansprache direkt einzugehen verändert sich nun der Stil der nächsten Mail von Seiten der Studentin - und zwar in Richtung einer Anpassung an das «Brief-Format» der Dozentin:

Studentin:

«Hallo Frau Bach, 
können Sie mir dann auch noch sagen, ob die folgende Seite auch nicht benutzt werden darf?

‘www.klusemann.onlinehome.des

Wenn Sie da auf «Surfen mit Frame` gehen, bekommen Sie auf der linken Seite Links angezeigt, wenn Sie dann auf Aktuelles gehen, kommen weitere Links (der erste, glaube ich, ist Unterrichtsstörungen).

Viele Grüsse

Tanja Bender»

In der Anschlusskommunikation wahrt die Dozentin weiter die Form, zieht sich nun aber aus konkreten Aspekten zurück und verweist auf Grundsätzliches:

Dozentin:

«Hallo Frau Bender,

ich möchte nochmals darauf verweisen, dass für die Bearbeitung des Begriffs die gleichen Kriterien gelten wie auch für Hausarbeiten und Referat. (...).

Beste Grüsse

Andrea Bach»

Die Botschaft scheint Gehör zu finden... Die studentische Folgemail ist erkennbar um die - wenn auch distanzierte - Erfüllung der Erwartungen des Gegenübers bemüht:

Studentin:

«Sehr geehrte Frau Bach,

ich habe den Begriff nochmal mit einer Literaturquelle überarbeitet (vgl. Anhang). Bitte schauen Sie es sich noch einmal an und sagen Sie mir, ob es nun so in Ordnung ist. Wenn ja, gebe ich es dann weiter an die Arbeitsgruppe. Viele Grüsse

Tanja Bender»

In diesem Beispiel werden Spiel- bzw. Schreibregeln - angesichts einer für die Dozentin anscheinend unbefriedigenden Ausgangssituation - explizit thematisiert, aber erst im Verlauf des weiteren Kommunikationsprozesses wird am Schreibstil der Studierenden deutlich, dass sie die als gesetzt erachteten Regeln der Briefform akzeptiert bzw. akzeptieren muss (und diese vermutlich bei Folgekontakten zu der Dozentin auch wieder aktivieren wird).

Es wird deutlich, dass sich im Hochschulkontext zwischen Dozierenden und Studierenden im Laufe der Zeit durchaus gemeinsame Normen in der E-Mail-Kommunikation entwickeln und etablieren können. Dabei variieren die Regeln sicher je nach der Beziehung der beteiligten Personen, nach ihrem Durchsetzungswillen 
bzw. ihrer Definitionsmacht sowie je nach Kontext, Anliegen bzw. Thema. Doch es bleibt jeweils ein Einzelfall; so bliebe abzuwarten, ob die Studentin Tanja Bender die etablierten Regeln auch auf den Mail-Verkehr mit anderen Hochschulangehörigen übertragen wird - und selbst wenn ..., nach ihr folgen für die Dozentin Andrea Bach weitere Generationen von Studierenden, mit denen die Normfrage erneut in vielen einzelnen Mails zu bearbeiten ist.

\section{$4 \quad$ Fazit}

Warum folgt die E-Mail-Kommunikation - etwa zwischen Studierenden und Lehrenden - kaum einheitlichen bzw. konsensfähigen Regeln? Warum wird die E-MailPraxis weiterhin problematisiert, obwohl das Medium schon seit vielen Jahren Bestandteil der gängigen Kommunikationspraxis an Hochschulen ist? Die geschilderten Überlegungen zum alltäglich auftretenden Phänomen der «Normverletzung» bei E-Mail-Texten lassen auf diese eingangs gestellten Fragen folgenden Antworten erkennen.

Sowohl die bisherige Forschung zur E-Mail als auch die genannten Textbeispiele aus dem Hochschulkontext machen deutlich, dass die Mail als Gattung - insbesondere zu Beginn der Schriftverkehrs - auf sehr unterschiedlichen Referenztextsorten fusst, die aus der mündlichen wie aus der schriftsprachlichen Kommunikation entlehnt werden. Die studentischen E-Mails werden also keineswegs regelfrei verfasst, sie bilden vielmehr die Vielfalt der sozialen wie kommunikativen Standpunkte der Verfasser/innen ab. Da die E-Mail von Studierenden eben nicht nur für die Kommunikation mit Hochschul-Lehrenden genutzt wird, sondern auch in vielen anderen privaten wie medialen Lebenskreisen präsent ist, bieten sich entsprechend heterogene Referenzen, um das Anliegen in der Auftaktkommunikation zu thematisieren (wie u. a. Brief, Chat-Eintrag, Kurznotiz, Alltagsgespräch oder Kommentar). Auch der wissenschaftliche Vergleich der E-Mail mit anderen Kommunikationsformaten führt die vielfältigen Erscheinungsformen von E-Mails vor Augen (vgl. u. a. Pansegrau 1997; Dürscheid 2009). Was eine «gute» E-Mail ist, liegt somit zunächst im Auge der Betrachterin bzw. des Betrachters. Die vielfältigen Interpretationen lassen sich dabei auf einem breiten Kontinuum verorten: von normativ traditionell (klassische Briefform wird präferiert) bis hin zu egalitär die Vielfältigkeit akzeptierend (mediale Möglichkeiten bestimmen die Form).

Diese Heterogenität trifft dann bei den Adressat/innen, hier also bei den Hochschul-Lehrenden, ebenfalls auf ein unterschiedliches Spektrum an Erwartungshaltungen - wiederum geprägt von verschiedenen Rollen- und Persönlichkeitsmerkmalen. Vom individuellen Standpunkt einer Dozentin oder eines Dozenten, in deren Beruf die Mail-Kommunikation inzwischen einen nicht unerheblichen Teil des Tagesgeschäfts ausmacht, wirkt die o. g. Varianz der elektronischen Ansprachefor- 
men jedoch verständlicherweise zum Teil irritierend, da zum einen die jeweilige Ausgangsreferenz einer Mail als unangemessene Verkehrsform im Hinblick auf den Kontext der Studierenden-Lehrenden-Beziehung an einer Hochschule erlebt werden kann - und da zum anderen die praktische Beantwortung der Mails aufgrund ihrer Verschiedenheit mit zusätzlichem Aufwand versehen ist.

Dass sich an dieser Konfliktlage der Heterogenität bei gleichzeitiger Normierungserwartung über die Jahre hinweg wenig geändert hat, kann in einem zweiten Schritt aus dem spezifischen Verlauf der E-Mail-Kommunikation als sozialer Praktik interpretiert werden, denn gemeinsame Normen entstehen gemäss den Annahmen des «social identity models» erst im Laufe der Interaktion, d. h. durch aktive Aushandlungsprozesse und deren Beobachtung durch die Beteiligten. So kann es zwar im Fall einer Mail-Sequenz zwischen Lehrenden und Studierenden durchaus zur (explizit thematisierten oder implizit übernommenen) Entwicklung von gemeinsamen Regeln für den Schriftverkehr kommen, doch gestaltet sich dies meist langwierig. Überdies bleibt das Regelungsergebnis an den meist dyadischen Einzelfall gebunden; d. h. da der E-Mail-Verkehr meist interpersonal geschlossen bleibt, kommt es nicht zu einer kollektiven Normbildung, die durch grössere Öffentlichkeiten dauerhaft geteilt würde.

Solange jedoch die Referenzformen zu Beginn einer E-Mail-Kommunikation derart unterschiedlich sind, solange die Erwartungshaltung bzw. die Reaktion der Adressat/innen ebenfalls einer heterogenen Praxis unterliegt, solange Normen dann ggf. in einem längeren Prozess innerhalb der jeweiligen digitalen Interaktion erst gemeinsam justiert werden müssen und dabei ohne Öffentlichkeitswirkung bleiben, solange bleibt die E-Mail als Textform wohl ein individualisierender «Selbstläufer» mit hohem Auslegungsreichtum und weiterhin bestehendem Konfliktpotenzial bezüglich der Erwartungen an eine «gute» Mail.

\section{Literatur}

Döring, Nicola. Sozialpsychologie des Internet. 2., vollst. überarb. und erw. Auflage. Göttingen: Hogrefe, 2003.

Dürscheid, Christa. "Merkmale der E-Mail-Kommunikation.» Von *hdl* bis * cul8r* - Sprache und Kommunikation in den Neuen Medien. Thema Deutsch, Bd. 7. Hrsg. v. Peter Schlobinski. Mannheim: Dudenverlag, 2006. 104-117.

Dürscheid, Christa. «E-Mail: eine neue Kommunikationsform?». Internet.kom. Neue Sprach- und Kommunikationsformen im WorldWideWeb. Band 1: Kommunikationsplattformen. Hrsg. v. Sandro M. Moraldo. Rom: Aracne Editrice, 2009. 21-53.

Emmer, Martin. Politische Mobilisierung durch das Internet? - Eine kommunikationswissenschaftliche Untersuchung zur Wirkung eines neuen Mediums. Bd. 22. München: Verlag Reinhard Fischer, 2005. 
Fischer, Martin; Gscheidle, Christoph. «Mitmachnetz Web 2.0: Rege Beteiligung nur in Communities. Ergebnisse der ARD/ZDF-Onlinestudie 2008.» Media Perspektiven 7 (2008): 356-364. http://www.daserste.de/service/studie08_4.pdf (27.5.2011).

Grigat, Felix. «Das E-Mail-Biest - und wie man es (vielleicht) im Zaum hält.» Forschung \& Lehre 9 (2010): 646.

Günther, Ulla; Wyss, Eva Lia. «E-Mail-Briefe - eine neue Textsorte zwischen Mündlichkeit und Schriftlichkeit.» Textstrukturen im Medienwandel. Hrsg. v. Ernest W. B. Hess-Lüttich, Werner Holly u. Ulrich Püschel. Frankfurt am Main: Lang, 1996. 61-86.

Hoeren, Thomas. «E-Mail-Gruselkabinett - 〈Hallöchen Herr Professor!»» SpiegelOnline 12.7.2006:

http://www.spiegel.de/unispiegel/wunderbar/0,1518,424426,00.html (27.5.2011).

Kesselheim, Wolfgang; Lindemann, Katrin. "Gemeinsam forschen lernen mit digitalen Medien: das Projekt ‘gi - Gesprächsanalyse interaktivı.» Digitale Medien für Lehre und Forschung. Hrsg. v. Schewa Mandel, Manuel Rutishauser u. Eva Seiler Schiedt. Münster: Waxmann, 2010. 106-117.

Kleinmann, Bernd; Özkilic, Murat; Göcks, Marc. «Studieren im Web 2.0.» HISBUS Kurzinformation Nr. 21. Studieren im Web 2.0. Hannover: HIS HochschullnformationsSystem, 2008. https://hisbus. his.de/hisbus/docs/hisbus21.pdf (27.5.11)

Linke, Angelika. «Informalisierung? Ent-Distanzierung? Familiarisierung? Sprach(gebrauchs)wandel als Indikator soziokultureller Entwicklungen.» Der Deutschunterricht 3 (2000): 66-77.

Mai, Jochen. «Sage und schweige - Regeln für die Kommunikation.» (2007): http:// karrierebibel.de/sage-und-schweige-kniggeregeln-fuer-die-kommunikation/ (27.5.2011).

Matzat, Uwe. "Soziale Netzwerke und die Entstehung von Normen in akademischen Online Gruppen.»(2002): http://umatzat.net/Soziale-Netzwerke-Normen.pdf (27.5.2011).

Meier, Jörg. «Vom Brief zur E-Mail - Kontinuität und Wandel.» Kommunikationsform E-Mail. Hrsg. v. Arne Ziegler u. Christa Dürscheid. Tübingen: Stauffenberg Verlag, 2002. 57-75.

Middendorff, Elke. "Computernutzung und Neue Medien im Studium.» Ergebnisse der 16. Sozialerhebung des Deutschen Studentenwerks (DWS). Bonn: Bundesministerium für Bildung und Forschung (2002): http://www.studentenwerke. de/se/2001/computernutzung.pdf (27.5.2011).

Pansegrau, Petra. "Dialogizität und Degrammatikalisierung in E-Mails.» Sprachwandel durch Computer. Hrsg. v. Rüdiger Weingarten. Weingarten: Opladen, 1997. 86-104. 
Postmes, Tom; Spears, Russell; Lea, Martin. «The Formation of Group Norms in Computer-Mediated Communication.» Human Communication Research. 26.3 (2007): 341-371.

Schäfers, Bernhard. Grundbegriffe der Soziologie. 5. Aufl.. Opladen: Leske \& Budrich, 1998.

Schelske, Andreas. "Computerunterstütze Vergesellschaftung.» Online Vergesellschaftung? - Mediensoziologische Perspektiven auf neue Kommunikationstechnologien. Hrsg. v. Michael Jäckel u. Manfred Mai. Wiesbaden: VS Verlag, 2005. 137-154.

Schmitz, Ulrich. «E-Mails kommen in die Jahre - Telefonbriefen auf dem Weg zur sprachlichen Kommunikation.» Kommunikationsform E-Mail. Hrsg. v. Arne Ziegler u. Christa Dürscheid. Tübingen: Stauffenberg Verlag, 2002. 33-56.

Schulmeister, Rolf. «Virtuelle Kommunikation: Editorial.» zeitschrift für e-learning 1, 1. Jg. (2006): 4-8.

http://www.e-learning-zeitschrift.org/01_2006/editorial.php (27.5.2011).

Schulmeister, Rolf. «Nachdenkliches zu Web 2.0 im Hochschulunterricht.» Statement zur Vorbereitung der Diskussionsrunde «Das Internet - Ein Bildungsraum?» anlässlich des EduCamps 2010 in Hamburg (2010):

http://blogs.epb.uni-hamburg.de/educamp/files/2010/01/Schulmeister_Nachdenkliches.pdf (27.5.2011).

Vedder, Ulrike. "Chance auf Verlangsamung? Was Briefe waren und werden können.» Forschung \& Lehre 9 (2010): 636-637.

Ziegler, Arne. «E-Mail - Textsorte oder Kommunikationsform? Eine textlinguistische Annäherung.» Kommunikationsform E-Mail. Hrsg. v. Arne Ziegler u. Christa Dürscheid. Tübingen: Stauffenberg Verlag, 2002. 9-32.

Zuber-Knost, Elisabeth. "Sie haben Post.» Informationsdienst Wissenschaft. Stabsabteilung Presse, Kommunikation und Marketing. Karlsruher Institute of Technology (29.7.2009):

http://idw-online.de/pages/de/news327727 (27.5.2011). 